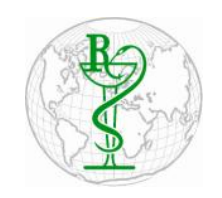

INDO GLOBAL JOURNAL OF

PHARMACEUTICAL SCIENCES

ISSN 2249- 1023

\title{
An Insight on the Emerging Regulations for Radiopharmaceuticals by IAEA, WHO and US
}

\author{
Shrutay Mehta, Dilip Maheshwari * \\ Department of Quality Assurance and Regulatory Affairs, L.J. Institute of Pharmacy, Ahmedabad-380020, India
}

\begin{abstract}
Address for
Correspondance

Dilip Maheshwari, dgmaheshwari

@gmail.com
\end{abstract}

Received:

13.12.2017

Accepted:

31.05 .2018

Keywords Radiopharmaceut icals;

Regulations; IAEA-WHO; US

\begin{abstract}
Radiopharmaceuticals are the radioactive substances or radioactive drugs for diagnostic or therapeutic interventions. The formulation of radiopharmaceuticals is different from ordinary pharmaceuticals. Many radiopharmaceuticals are released and administered to patients shortly after their production because radiopharmaceuticals may decompose during process and labeling steps due to variation in environmental conditions, indicating serious stability concerns. So, administrative prerequisites must be taken for the formulation of the radiopharmaceuticals. Radiopharmaceuticals production, unlike conventional pharmaceuticals production, is still raw and processing at a full fledged speed. Hence, implementing the cGMP guidelines which are applicable for the drugs industry is both difficult and expensive. The present article introduces radiopharmaceuticals in an effective and useful way. Herein, the radiopharmaceuticals are described as a trustworthy aid for curing the life threatening diseases. It demonstrates a brief investigation of the guidelines constituted by the regulatory bodies for the radiopharmaceuticals. It emphasizes on understanding the mandatory regulations to be taken for the radiopharmaceuticals in various countries. It includes the regulations framed for radiopharmaceuticals by IAEA-WHO and US. The present work provides information on the guidelines used by the International bodies and US for the regulation of radiopharmaceuticals by the special committees. The regulations in US are stringent whereas, in several countries, the regulations are still to get illumination. It withholds the names of the guidelines in the world to be alluded for the radiopharmaceuticals and the regulatory bodies responsible for the regulation. (c) 2018 iGlobal Research and Publishing Foundation. All rights reserved.
\end{abstract}

Cite this article as: Mehta, S.; Maheshwari, D. An insight on the emerging regulations for radiopharmaceuticals by IAEA, WHO and US. Indo Global Journal of Pharmaceutical Sciences, 2018; 8(3): $80-87$.

\section{INTRODUCTION}

\section{Radiopharmaceuticals [1-4]}

a) A drug is generally chemical substance which provides biological effects on humans as well as on animals. In the broadcast of pharmacology term; drug is defined as a chemical substance used for diagnosis of disease or to enhance mental or physical well-being.

b) Drugs are producing a physiological effect when ingested or otherwise introduced into the body. c) A radiopharmaceutical is a pharmaceutical formulation containing radioactive compound which is used for the diagnosis and therapeutic treatment of human diseases.

d) In designing a radiopharmaceutical, a pharmaceutical is first chosen on the basis of its preferential localization in a given organ or its participation in the physiologic function of the organ. Then a suitable radionuclide is tagged onto the chosen pharmaceutical such that after administration of the 


\section{Indo Global Journal of Pharmaceutical Sciences, 2018; 8(3): 80-87}

radiopharmaceutical, radiations emitted from it are detected by a radiation detector.

e) Once administered to the patient they can localize to specific organs or cellular receptors.

\section{Classification of Radiopharmaceuticals}

According to WHO, radiopharmaceuticals can be classified into four categories as follows:

1. Radiopharmaceutical preparation

2. Radionuclide generator

3. Radiopharmaceutical precursor

4. Kit for radiopharmaceutical preparation

Examples of Radiopharmaceuticals

1. Fluorine-18 FDG (Neuraceq ${ }^{\mathrm{TM}}$ )

Used in positron emission tomography imaging.

2. Iodine-125 human serum albumin (Jeanatope, Isojex)

Used in plasma volume determinations.

3. Molybdenum Mo-99 generator (DRYTEC ${ }^{\mathrm{TM}}$, Technelite ${ }^{\circledR}$, UltraTechneKow ${ }^{\mathrm{TM}} \mathrm{V} 4$ )

Used in generation of Tc-99m sodium pertechnetate.

4. Technetium-99m exametazine (Ceretec ${ }^{\mathrm{TM}}$ )

Used in cerebral perfusion imaging.

5. Thallium-201 chloride (DuPont)

Used in myocardial perfusion imaging and parathyroid \& tumor imaging.

\section{Regulations for Radiopharmaceuticals [5]}

- Regulatory agencies are established to protect the public health and usually communicate with commercial pharmaceutical companies who have dedicated staff for this process.

- Non-commercial manufacturers should be aware of the specialist knowledge and language required in these communications and account for that in their approach, seeking expert advice or training individuals for this role.

- The specific requirements i.e. facilities and procedures for the production, use, and storage of radiopharmaceuticals are subject to licensing by national and/or regional authorities.

- This licensing includes compliance both with regulations governing pharmaceutical preparations and with those governing radioactive materials.

- The offices and techniques for the generation, use, and capacity of radiopharmaceuticals are liable to authorizing by national and/or provincial powers.

- Regulations governing radioactive materials include those on safe handling and production of radioisotopes, they are:

International Basic Safety Standards for Protection against Ionizing Radiation and for the Safety of Radiation Sources
(IAEA, Vienna, 2003, CD-ROM Edition) Safety Series No. $115 / \mathrm{CD}$

$\square$ Radiological Protection for Medical Exposure to Ionizing Radiation Safety Guide (IAEA, Vienna, 2002) Safety Standard Series No. RSG-1.5.

Guidelines on Good Manufacturing Practices for radiopharmaceutical products (WHO Technical Report Series, No. 908, 2003) Annex 3.

\section{United States: [6-8]}

- The Food and Drug Administration (FDA or USFDA) is a federal agency of the United States Department of Health and Human Services, one of the United States federal executive departments.

- Drugs, whose effect is chemical, such as the recent cancer therapeutic Ra-223 (Xofigo), all of the imaging drugs and biologics, which occur naturally within the human body, such as antibodies are licensed are approved by Center for Drug Evaluation and Research (CDER).

- Some radiopharmaceuticals are considered medical devices because their effect is considered physical, such as Y-90 labeled microspheres for liver cancer. These are approved by Center for Devices and Radiological Health (CDRH).

- The standard for the manufacture of radiopharmaceuticals ('drugs') in the United States is 21 CFR 212. This standard applies to approve PET radiopharmaceuticals as well as PET radiopharmaceuticals in phase III and IV clinical trials.

- PET radiopharmaceuticals being produced for earlier phase clinical trials have the option to be produced using either the standards within 21 CFR 212 or chapter $<823\rangle$ of the United States Pharmacopeia (USP, 32nd edition, 2009).

- There is another Federal Agency that is currently authorized to regulate the routine use of radioactive drugs from the standpoint of reducing unnecessary radiation exposure to patients, Nuclear Regulatory Commission (NRC).

- All NRC regulations are published in the Federal Register and codified in Title 10, Chapter 1, of the Code of Federal Regulations (CFR).

- The regulations that most directly affect radiopharmaceutical practice are contained in:

10 CFR 35 (Human Uses of Byproduct Material)

10 CFR 20 (Standards for Protection Against Radiation)

10 CFR 71 (Packaging and Transportaion of Radioactive Materials)

\section{Summary}

1. Regulations for radiopharmaceuticals by IAEA and WHO [9-10]

A. IAEA 


\section{Indo Global Journal of Pharmaceutical Sciences, 2018; 8(3): 80-87}

The International Atomic Energy Agency (IAEA) is an international organization that seeks to promote the peaceful use of nuclear energy, and to inhibit its use for any military purpose, including nuclear weapons. The IAEA was established as an autonomous organization on 29 July

1957. Though established independently, the IAEA, reports to both the United Nations General Assembly and Security Council. The IAEA serves as an intergovernmental forum for scientific and technical cooperation in the peaceful use of nuclear technology and nuclear power worldwide. The programs of the IAEA encourage the development of the peaceful applications of nuclear technology, provide international safeguards against misuse of nuclear technology and nuclear materials, and promote nuclear safety (including radiation protection) and nuclear security standards and their implementation. The IAEA has its headquarters in Vienna.

\section{B. WHO}

The World Health Organization (WHO) is a specialized agency of the United Nations that is concerned with international public health. It was established on 7 April 1948, headquartered in Geneva, Switzerland. The constitution of the World Health Organization had been signed by 61 countries on 22 July 1946, with the first meeting of the World Health Assembly finishing on 24 July 1948. Since its creation, it has played a leading role in the eradication of smallpox. Its current priorities include communicable diseases, in particular HIV/AIDS, Ebola, malaria and tuberculosis; the mitigation of the effects of non-communicable diseases; sexual and reproductive health, development, and aging; nutrition, food security and healthy eating; occupational health; substance abuse; and driving the development of reporting, publications, and networking.

\subsection{Definition of Radiopharmaceuticals}

Radiopharmaceuticals are unique medicinal formulations containing radioisotopes which are used in major clinical areas for diagnosis and/or therapy.

Radiopharmaceuticals are radiolabelled compounds designed to deliver diagnostic information as a result of their incorporation with selected cellular targets. These exquisite molecular tools are essential components of nuclear medicine technology and must be prepared prior to administration to the patient. The production of radiopharmaceuticals must be performed by a licensed commercial organization, or alternatively using in-house good manufacturing practice (GMP) compliant facilities.

\subsection{Good Manufacturing Practices for Radiopharmaceuticals[11]}

1.2.1 Quality assurance and Quality control

- Radiopharmaceuticals are almost constantly utilized before all quality control testing (e.g. tests for sterility, endotoxin, radionuclidic purity, and so on.) has been finished. The usage of and consistence with the quality confirmation program are in this way basic.

- Quality confirmation as well as quality control ought to have the accompanying chief duties:

a) the preparation of detailed instructions for each test and analysis; ensuring the adequate identification and segregation of test samples to avoid mix-ups and cross-contamination;

b) ensuring that environmental monitoring and equipment and process validation are conducted as appropriate for evaluating the adequacy of the manufacturing conditions;

c) the release or rejection of starting materials and intermediate products; the release or rejection of packaging and labelling materials;

d) the release or rejection of each batch of finished preparation;

e) the evaluation of the adequacy of the conditions under which the starting materials, intermediate products and finished radiopharmaceutical preparations are stored;

f) the evaluation of the quality and stability of the finished products and, when necessary, of the starting materials and intermediate products;

g) the establishment of expiry dates on the basis of the validity period related to specified storage conditions;

h) the establishment and revision of the control procedures and specifications;

i) assuming the responsibility for retaining samples of radiopharmaceutical products;

j) assuming the responsibility for keeping adequate records of the distribution of the radiopharmaceutical products.

Whenever the measure of the foundation licenses, quality confirmation and quality control obligations ought to be sorted out in particular gatherings. Quality confirmation ought to likewise incorporate the checking and approval of the generation procedure.

A producer's quality control research center ought to be isolated from the creation region. The control research center ought to be outlined, prepared and of such a size as to be an independent element, with sufficient arrangement for the capacity of archives and tests, the readiness of records and the execution of the vital tests. 


\subsubsection{Handling}

- It is necessary to mark the area in which the radio active work is carried out and it should be monitored regularly at periodic intervals.

- Unnecessary developments of people or materials ought to be maintained a strategic distance from in the hot research facility or radiopharmacy.

- All the radiation specialists must wear appropriate defensive dress and radiation observing gadgets.

- Surgical gloves are important. At the point when not being used the radionuclides must be kept in fixed compartments. The zone ought to be studied consistently for both pollution and introduction perils.

- Do not pipette by mouth. Work zones ought to be secured with a plastic glass or stainless plate with retentive paper ought to be utilized to get any spills and to keep the spread of contamination. The radiation overview meter ought to be utilized to guarantee the wellbeing of laborers and open, review and wipe test recommended activity levels.

\subsubsection{Packaging and labelling}

Every radiopharmaceutical preparation must comply with the labelling requirements established under Good Manufacturing Practice. The label on the primary container should include:

- A statement that the product is radioactive or the international symbol for radioactivity.

- The name of the radiopharmaceutical preparation; where appropriate, that the preparation is for diagnostic or for therapeutic use.

- The route of administration; the total radioactivity present at a stated date and, where necessary, time.

- For solutions, a statement of the radioactivity in a suitable volume (for example, in $\mathrm{MBq}$ per $\mathrm{ml}$ of the solution) may be given instead.

- The expiry date and, where necessary, time; the batch (lot) number assigned by the manufacturer; for solutions, the total volume.

The label on the outer package should include:

- A statement that the product is radioactive or the international symbol for Radioactivity the name of the radiopharmaceutical preparation; where appropriate, that the preparation is for diagnostic or for therapeutic use.

- The route of administration; the total radioactivity present at a stated date and, where necessary, time; for solutions, a statement of the radioactivity in a suitable volume (for example, in $\mathrm{MBq}$ per $\mathrm{ml}$ of the solution) may be given instead.

- The expiry date and, where necessary, time.
- The batch (lot) number assigned by the manufacturer; for solutions, the total volume; any special storage requirements with respect to temperature and light.

- Where applicable, the name and concentration of any added microbial preservatives or, where necessary, that no antimicrobial preservative has been added.

\subsubsection{Storage}

- Radiopharmaceuticals should be kept in well-closed containers and stored in an area assigned for the purpose.

- The storage conditions should be such that the maximum radiation dose rate to which persons may be exposed is reduced to an acceptable level.

- Care should be taken to comply with national regulations for protection against ionizing radiation.

- Radiopharmaceutical arrangements that are expected for parenteral utilize ought to be kept in a glass vial, ampoule or syringe that is adequately straightforward to allow the visual investigation of the substance. Glass holders may obscure under the impact of radiation.

\section{Regulations for radiopharmaceuticals by US 2.1 Definition of Radiopharmaceutical [12]}

An article that is intended for use in the diagnosis or monitoring of a disease or a manifestation of a disease in humans and that exhibits spontaneous disintegration of unstable nuclei with the emission of nuclear particles or photons; or Any nonradioactive reagent kit or nuclide generator that is intended to be used in the preparation of such article as defined above.

\subsection{Good Manufacturing Practices for Radiopharmaceuticals[13]}

2.2.1 Laboratory Controls

It requires the establishment and implementation of procedures for testing components, in- process materials, and finished radiopharmaceuticals. All necessary tests of materials and products must be documented. Each laboratory must have sampling and testing procedures designed to ensure that components, drug product containers and closures, in process materials, and radiopharmaceuticals conform to appropriate standards. Analytical methods and test equipment must be suitable for their intended uses.

Reagents, arrangements, and supplies utilized as a part of testing strategies must be satisfactorily controlled. The preventive support, alignment, and methodology to ensure 


\section{Indo Global Journal of Pharmaceutical Sciences, 2018; 8(3): 80-87}

that the hardware is working appropriately should be reported. An entire record of all tests identified with the generation of the radiopharmaceuticals must be kept to guarantee consistence with set up particulars and models, including examinations and measures, as takes after:

- An appropriate recognizable proof of the specimen.

- A portrayal of every technique utilized as a part of the testing of the specimen, a record of all counts performed regarding each test, and an announcement of the weight or estimation of the example utilized for each test.

- A finish record of all information (counting diagrams, outlines, and spectra), e.g., a print- out of the chromatogram with the figured measures of every segment broke down by the test.

- A proclamation of consequences of the tests and their connection to acknowledgment criteria.

- The initials or mark of the investigator and the date of the test.

\subsubsection{Stability Testing}

- It requires the foundation of a composed security testing program for every radiopharmaceutical.

- Radiopharmaceutical molecules are expected to remain stable during storage. Although radiopharmaceuticals have extremely short shelf lives, because of their short half-lives compared to other kinds of drug products, there are stability concerns due to radiation- related radiolysis.

- Certain radiopharmaceuticals (e.g., F 18 fluorodopa) can undergo very rapid chemical changes. Therefore, appropriate parameters should be evaluated to establish and document the stability of radiopharmaceuticals under proposed storage conditions. Examples of stability parameters include radiochemical identity and purity (including levels of radiochemical impurities), appearance, $\mathrm{pH}$, stabilizer or preservative effectiveness, and chemical purity.

- The officials recommend that appropriate stabilityindicating methods that can distinguish degradation products and impurities be used.

- Stability testing of the radiopharmaceutical should be performed at the highest radioactive concentration, and the whole batch volume in the intended container/closure should be stored.

- At minimum three generation keeps running of the last item ought to be considered for a day and age equivalent to the marked time span of usability of the radiopharmaceutical.

In spite of the fact that strength thinks about in support of a termination dating period are required for endorsement of a radiopharmaceutical, resulting changes to the close date could be made without earlier endorsement (changes would be noted in the yearly report for the medication item).

- The stability studies supporting a changed expiration date should be conducted in accordance with the postapproval stability protocol in the drug product's approved application.

\subsubsection{Radiation Safety Assessment[14]}

\subsubsection{General Considerations}

It is recommended that the sponsor should submit sufficient data from animal or human studies to allow a reasonable calculation of radiation absorbed dose to the whole body and to critical organs upon administration to a human subject. At a minimum, we recommend that the following organs and tissues be included in dosimetry estimates: (1) all target organs/tissues; (2) bone; (3) bone marrow; (4) liver; (5) spleen; (6) adrenal glands; (7) kidney; (8) lung; (9) heart; (10) urinary bladder; (11) gall bladder; (12) thyroid; (13) brain; (14) gonads; (15) gastrointestinal tract; and (16) adjacent organs of interest. When a radiopharmaceutical is being developed for pediatric use, it may be appropriate to evaluate the radiation absorbed dose in all organs, rather than in selected organs. Moreover, we recommend that organ dosimetry be estimated for the pediatric age groups (e.g., neonates, infants, children, adolescents) in which the radiopharmaceutical is intended to be used.

It is advisable that the amount of radiation delivered by internal administration of radiopharmaceuticals be calculated by internal radiation dosimetry.

It becomes that the methodology used to assess radiation safety be specified including reference to the body models that were used. The mathematical equations used to derive the radiation doses and the absorbed dose estimates are provided along with a full description of assumptions. Further it is recommended that sample calculations and all pertinent assumptions be listed and submitted. The reference to the body, organ, or tissue

model used in the dosimetry calculations be specified, particularly for new models being tested.

It recommended that safety hazards for patients and health care workers during and after administration of the radiolabeled product be identified, evaluated, and managed appropriately.

2.2.3.2 Calculation of Radiation Dose to the Target Organs or Tissues

For established radionuclides used as diagnostic agents (e.g., TC-99m, IN-111), the following items be determined based on the average patient: 


\section{Indo Global Journal of Pharmaceutical Sciences, 2018; 8(3): 80-87}

- The amount of radioactivity that accumulates in the target tissue(s) or organ(s)

- The amount of radioactivity that accumulates in tissues adjacent to the target tissue(s) or organ(s)

- The residence time of the radiopharmaceutical in the target tissue(s) or organ(s) and in adjacent regions

- For new radionuclide diagnostic agents, consult the appropriate review division.

\subsubsection{Maximum Absorbed Radiation Dose}

It is recommended that the amount of radioactive material administered to human subjects be the smallest radiation dose practical to perform the procedure without jeopardizing the benefits obtained.

It says that calculations anticipate possible changes in dosimetry that might occur in the presence of diseases in organs that are critical in metabolism or excretion of the radiopharmaceutical. For example, renal dysfunction may cause a larger fraction of the administered dose to be cleared by the hepatobiliary system (or vice versa).

This depicts that possible changes in dosimetry resulting from patient-to-patient variations in antigen or receptor mass be considered in dosimetry calculations. For example, a large tumor mass may result in a larger-thanexpected radiation dose to a target organ from a diagnostic radiopharmaceutical that has specificity for a tumor antigen.

It is recommended that the mathematical equations used to derive the estimates of the radiation dose and the absorbed dose be provided along with a full description of assumptions that were made. Also, the sample calculations and all pertinent assumptions be listed.

It is recommended that calculations of dose estimates be performed assuming freshly labeled material (to account for the maximum amount of radioactivity) as well as the maximum shelf life of the diagnostic radiopharmaceutical (to allow for the upper limit of radioactive decay contaminants). Other calculations recommended are:

- Radiation doses from x-ray procedures that are part of the study (i.e., would not have occurred but for the study) should also be included. The possibility of follow-up studies should be considered for inclusion in the dose calculation.

-Be expressed as gray (Gy) per megabecquerel (MBq) or per millicurie $(\mathrm{mCi})$ of radionuclide
Be presented in a tabular format and include doses of individual absorbed radiation for the target tissues or organs.

\subsubsection{Quality Control}

- It requires that sterility testing be begun inside 30 hours after the fulfillment of radiopharmaceutical generation; be that as it may, the 30 -hour prerequisite might be surpassed because of an end of the week or occasion.

- The regulation states that if the sample for sterility testing is held longer than indicated (e.g., over the weekend), the radiopharmaceutical producer must demonstrate that the longer period does not adversely affect the sample and the test results obtained will be equivalent.

- The samples should be stored appropriately (e.g., under refrigeration). Verification of equivalent results can be accomplished by inoculation of a USP indicator organism (e.g., E. coli) and should demonstrate that there is little, if any, loss in viability of the inoculated microorganism. - Testing be directed in a controlled range, for example, a LAFW with clean-room clothing. Aseptic procedures ought to be utilized for sterility testing. The most serious danger of false positive outcomes emerges in the inspecting and exchange of the test aliquot from the vial to the media. It might be advantageous to apply coordinate immunization into business media.

- The media should be observed on days 3, 7, and 14 after inoculation, but it is prudent to observe the media more often during the first week of incubation. The USP Bacterial Endotoxins Test (BET) should be performed for a sterile radiopharmaceutical product that is intended for injection. The BET contains gel-clot and rapid photometric methods for endotoxin measurement. The product can be distributed under control after a pharmacopeial bacterial endotoxin test is initiated. However, the endotoxin results should meet the acceptance criteria before administering the product to humans. If the result of any bacterial endotoxin test exceeds the acceptance limit, or if a sterility test is positive for microbial growth, a complete investigation be conducted immediately and documented and corrective actions based on the results of the investigations be implemented promptly.

\subsubsection{Quality Assurance}

In the controls, the accompanying exercises are characterized as the obligations of the quality confirmation work:

- Oversee generation operations to guarantee that radiopharmaceutical have satisfactorily characterized personality, quality, quality, and virtue. 
Indo Global Journal of Pharmaceutical Sciences, 2018; 8(3): 80-87

- Examine and approve or reject components, containers, closures, in-process materials, packaging materials, labeling, and finished dosage forms to ensure that all these meet their current specifications.

- Approve or reject specifications, methods, processes, and procedures, and any proposed changes.

- Review production records to determine whether errors occurred. If errors have occurred or specifications have not been met, determine the need for an investigation and take appropriate corrective action.

- The quality assurance function in a radiopharmaceutical production facility typically consists of execution and oversight activities.

It is recommended that the execution of quality assurance functions include the following:

- Examine and evaluate each lot of incoming material before use to ensure that the material meets its established specifications.

- Review the production batch records and laboratory control records for accuracy, completeness, and conformance to established specifications before authorizing the final release or rejection of a batch or lot of a radiopharmaceutical.

- Ensure that deviations from normal procedures are documented and justified.

It is recommended that the oversight of quality assurance functions include the following:

- Approve procedures, specifications, process, and methods.

- Ensure that personnel are properly trained and qualified, as appropriate.

- Ensure that radiopharmaceuticals have adequately defined identity, strength, quality, and purity.

- Ensure that all errors are reviewed. When it is determined that an investigation is appropriate, document the investigation and take corrective action(s) to prevent the recurrence of the errors.

- Conduct periodic audits to monitor compliance with established procedures and practices. For radiopharmaceutical production facilities currently producing one or two radiopharmaceutical, employees located at the facility can perform both the daily execution and oversight functions. On the other hand, a commercial radiopharmaceutical firm managing multiple production facilities may choose to have an entity located outside the radiopharmaceutical production facility help to achieve the objective of production oversight and more efficient management. For example, a corporate quality assurance/quality control department, or consultants, can provide oversight.
2.2.6 Labelling and Packaging

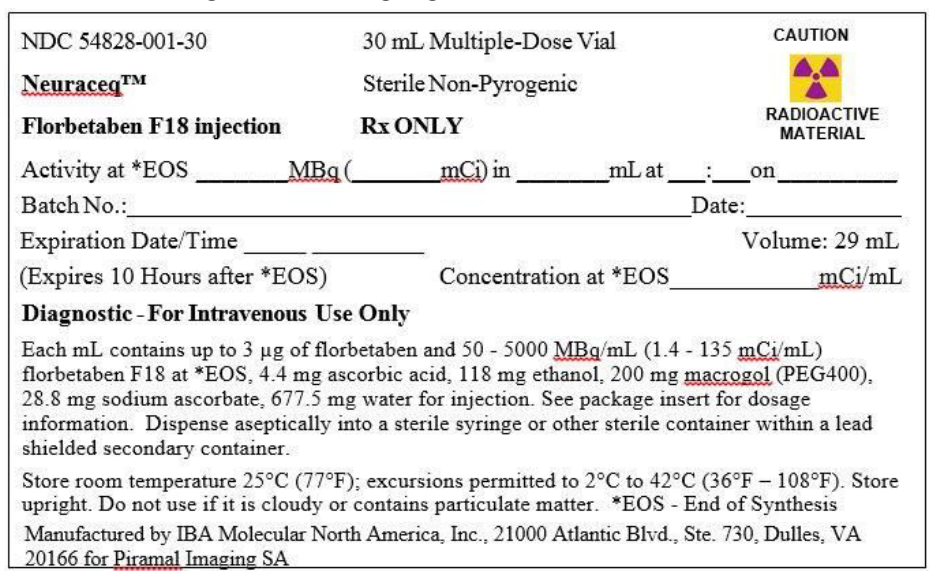

Fig.1: Example of radiopharmaceutical label[15]

- It must ensure that each container of licensed material is labeled with the radiation symbol and the words CAUTION (or DANGER), RADIOACTIVE MATERIAL.

- The label must also provide sufficient information (such as radionuclide and quantity of radioactivity at a specified date and time) to permit individuals handling or using the containers or working in the vicinity of the containers to take precautions to avoid or minimize exposures.

- It must remove or deface the radioactive material label or otherwise clearly indicate that the container no longer contains radioactive materials before removal or disposal of empty uncontaminated containers to unrestricted areas.

- Radiopharmaceuticals intended for commercial distribution radioactive drugs containing byproduct material must satisfy the following two labeling requirements:

(1) A label must be affixed to each transport radiation shield and include the radiation symbol and the words CAUTION (or DANGER), RADIOACTIVE MATERIAL, the name of the radioactive drug, and the quantity of radioactivity at a specified date and time; and

(2) A label must be affixed to each syringe, vial, or other container and include the radiation symbol and the words CAUTION (or DANGER), RADIOACTIVE MATERIAL, and an identifier that ensures that the container can be correlated with the information on the transport radiation shield label.

- Each syringe or vial that contains unsealed byproduct material must be labeled to identify the radioactive drug. Each syringe shield and vial shield also must be labeled, unless the label on the syringe or vial is visible when shielded. 


\section{Indo Global Journal of Pharmaceutical Sciences, 2018; 8(3): 80-87}

\section{CONCLUSION}

Radiopharmaceuticals are more precise dosage form and require to have limited exposure for the safety of the patients. As they are used in the life threatening diseases, no misconduct must be observed in the application of radiopharmaceuticals. Moreover, radiopharmaceuticals should be considered as the most regulated materials administered to patients because they are controlled both as drugs and as radioactive substances. Hence, the regulation for radiopharmaceuticals is compulsory, as it is used in life threatening conditions. Also, the regulations framed, help for the better manufacturing and administration of the radiopharmaceuticals. The guidelines framed, itself, shows the need and the importance for the regulation of the radiopharmaceuticals in the world. By providing with the regulations, the awareness about the effectiveness and usefulness of radiopharmaceuticals can also be brought to the limelight.

\section{ACKNOWLEDGEMENTS}

The authors are thankful to Dr. K. Pundarikakshudu, director of L. J. Institute of Pharmacy, Ahmedabad, India for providing all the facilities to carry out work.

\section{REFERENCES}

1. Allen LV., Ansel HC. Ansel's Pharmaceutical Dosage Forms and Drug Delivery Systems, 10th Edn; Lippincott Williams \& Wilkins: Philadelphia, 2014.

2. Saha GB. Fundamentals of Nuclear Pharmacy, 5th Edn; Springer-Verlag New York: New York, 2004.

3. World Health Organization, "Guidelines on Good Manufacturing Practices for radiopharmaceutical products",2003,http://apps.who.int/medicinedocs/documents/s2011 1en/s20111en.pdf (Last Accessed Date 13/01/2017)

4. International Atomic Energy Agency, "Radiopharmaceuticals: Production and Availability",https://www.iaea.org/About/Policy/GC/GC51/GC51I nfDocuments/English/ gc51inf-3-att2_en.pdf (Last Accessed Date $15 / 01 / 2017)$

5. World Health Organization, "Radiopharmaceuticals", November 2008, http://www.who.int/medicines/publications/pharmacopoeia/Radge nmono.pdf (Last Accessed Date 16/01/2017)

6. International Atomic Energy Agency, "Good Practice for Introducing Radiopharmaceuticals for Clinical Use”, October 1956,http://www- pub.iaea.org/MTCD/Publications/PDF/TE-

1782_web.pdf (Last Accessed Date 16/01/2017)

7. Nuclear Regulatory Commission, "History of NRC",http://www.nrc.gov/about- nrc/history.html\#blogs (Last Accessed Date 18/01/2017)

8. Center for Devices and Radiological Health, "Functions of CDRH", http://www.fda.gov/Training/CDRHLearn/default.html (Last Accessed Date 18/01/2017)

9. International Atomic Energy Agency,

"Overview of IAEA",

https://www.iaea.org/about/overview/ (Last Accessed Date 21/01/2017)

10. WHO, "Introduction about WHO", http://www.who.int/about/en/ (Last Accessed Date 23/01/2017)

11. WHO technical report series, "Guidelines on Good Manufacturing Practices for radiopharmaceutical products", 2003

http://apps.who.int/medicinedocs/documents/s20111en/s20111en.p df (Last Accessed Date 24/01/2017)

12. USFDA, "Subpart-D

Radiopharmaceuticals", 2016,

http://www.accessdata.fda.gov/scripts/cdrh/cfdocs/cfcfr/CFRSearc h.cfm?CFRPart=601\& showFR=1\&subpartNode=21:7.0.1.1.2.4 (Last Accessed Date 26/01/2017)

13. Guidances, "PET Drugs - Current Good Manufacturing Practice (CGMP)", August 2011,

http://www.fda.gov/downloads/Dmgs/GuidanceComplianceRegula toryInformation/Guida nces/UCM266640.pdf (Last Accessed Date 28/01/2017)

14. Guidance for Industry- conducting safety assessments, "Medical Imaging Drug and Biological Products", May 2003, http://www.fda.gov/downloads/Drugs/Guidances/ucm071600.pdf (Last Accessed Date 31/01/2017)

15. Image of label, "Example of radiopharmaceutical",

https://www.google.co.in/search?q=labelling+of+radiopharmaceuti cals + as +per+USFDA\&espv $=2 \& b i w=1164 \& b i h=586 \&$ source $=\ln m$ s\&tbm $=$ isch\&sa $=X \& v e d=0$ ahUKEwjDyea5 huvRAhWLuY8KHbHiANIQ_AUIBygC\#tbm=isch\&q=labelling+ of+neuraceq+radiopha rmaceuticals+as+per+US\&imgrc $=x j E V 8 W X 1 j R Y 6 I M \% 3 \mathrm{~A}$ (Last Accessed Date 02/02/2017)

Indo Global Journal of Pharmaceutical Sciences( ISSN 22491023 ; UGC Journal No.: 44477; CODEN- IGJPAI; NLM ID: 101610675) indexed and abstracted in EMBASE(Elsevier), UGC Journal List, National Library of Medicine (NLM) Catalog, Elsevier( EMBASE), ResearchGate, Publons, CAS (ACS), Index Copernicus, Google Scholar and many more. For further details, visit http://iglobaljournal.com 